\title{
INCLUSION, CONTRAST AND POLYSEMY IN DICTIONARIES: THE RELATIONSHIP BETWEEN THEORY, LANGUAGE USE AND LEXICOGRAPHIC PRACTICE
}

\author{
ANU KOSKELA \\ De Montfort University, Leicester \\ akoskela@dmu.ac.uk
}

\begin{abstract}
This paper explores the lexicographic representation of a type of polysemy that arises when the meaning of one lexical item can either include or contrast with the meaning of another, as in the case of dog/bitch, shoe/boot, finger/thumb and animal/bird. A survey of how such pairs are represented in monolingual English dictionaries showed that dictionaries mostly represent as explicitly polysemous those lexical items whose broader and narrower readings are more distinctive and clearly separable in definitional terms. They commonly only represented the broader readings for terms that are in fact frequently used in the narrower reading, as shown by data from the British National Corpus.
\end{abstract}

Keywords: semantics, lexicology, lexicography, polysemy

\section{Introduction}

Most modern dictionaries aim to record and describe the meanings of lexical forms as used in a given language community and do so in such a way that the dictionary user may understand how the words can be used (Zgusta 1971). However, given the inherent flexibility of word meaning, it is not always obvious when particular usages should be enumerated as separate senses or how the relationship between particular lexical units should be represented. This paper focuses on a particular type of meaning variation as a way of exploring the relationship between lexicographic practice, language use and theoretical accounts of word meaning.

The focus here are cases where two lexical items have a 'dual' relationship whereby the meaning of one term can be construed more broadly or narrowly, so that it either includes or contrasts with the meaning of the other. This is illustrated by $d o g$, whose general 'canine mammal' reading is hyperonymous to bitch, while the more specific 'male canine mammal' reading is a co-hyponym of bitch. A similar relationship holds between shoe and boot, as illustrated by B's alternative answers in 1.

(1) A: Are you going to wear shoes?

B1: Yes - I'm not going out barefoot!

B2: No, it's raining. I'll wear my boots. 
Other similar pairs with a dual relationship include finger/thumb, cow/bull, animal/bird, plant/tree and cup/mug. The lexical item with the broader and narrower readings may be called an autohyponym (Horn 1984) or, alternatively, a vertical polyseme (alluding to the common practice of representing the relationship of hyponymy on the vertical axis in diagrams). ${ }^{1}$ For ease of reference, I will here use the general label A-term for any lexical item with broader and narrower readings (e.g. dog, shoe, finger etc.) and B-term for the lexical item that can either function as a hyponym or a co-hyponym of the A-term (e.g. bitch, boot, thumb etc.). The abbreviation $A l$ refers to the broader reading (e.g. $\operatorname{dog}$ 'canine mammal', shoe 'item of footwear', finger 'one of the hand digits') and $A 2$ to the narrower reading ('male canine mammal', 'item of footwear that reaches only to the ankle', 'one of the hand digits other than the thumb').

Although the term vertical polysemy implies that the A1 and A2 readings are distinct senses, sense demarcation in such cases is a vexed issue from both theoretical and lexicographic perspectives. Because the narrower reading can always be subsumed under the broader one, some traditional ambiguity criteria such as the definitional criterion or the identity-of-sense test are not applicable (Zwicky \& Sadock 1975; Geeraerts 1993). Furthermore, in many cases of vertical polysemy the distinction between the A1 and A2 readings is very subtle. Consequently some have argued against viewing them as distinct senses, preferring instead to account for the meaning variation through pragmatic means (e.g. Becker 2002; Huang 2009) or as a reflection of the flexibility of prototype category boundaries (Lehrer 1990a). However, the term polysemy can be justified on an account where the distinction between ambiguity and vagueness is seen as a matter of degree (e.g. Tuggy 1993; Croft \& Cruse 2004). Viewed from this perspective, vertical polysemes whose broader and narrower readings are only subtly distinctive would fall somewhere between full ambiguity and vagueness. However, it is important to note that in principle even very subtly distinctive readings may nevertheless be conventionalised and established as distinct senses, as a function of their frequency in language use (Rohdenburg 1985a).

Such potential variation in the distinctness and conventionality of the broader and narrower readings makes the lexicographic representation of vertical polysemes a challenging issue. It involves not only the question of when the A1 and A2 readings should be enumerated as separate senses, but also whether the A/B relationship should be represented as one of inclusion or contrast. To explore different dictionaries' practices, I surveyed the representation of 12 pairs of A- and B-terms (including shoe/boot, finger/thumb, plant/tree etc.) in seven desk-size monolingual English dictionaries. The survey showed that dictionaries tend to represent as explicitly vertically polysemous those terms whose broader and narrower readings are more distinctive and clearly separable in definitional terms. On the other hand, they commonly only represented the broader A1 readings for terms that are in fact frequently used in the

\footnotetext{
${ }^{1}$ Autohyponymy or vertical polysemy is not restricted to cases where a word has a dual relationship with another word. The verb drink, for instance, has a broader 'consume liquid' and a narrower 'consume alcohol' reading, and is therefore an autohyponym/vertical polyseme. However, its narrower reading is not defined in contrast with another word and instead represents a salient subset of the class designated by the broader reading. Horn's (1984) neoGricean pragmatic account of autohyponymy maintains that different types of autohyponyms are motivated by different pragmatic principles.
} 
narrower A2 reading, as shown by data from the British National Corpus (BYU-BNC, Davies 2004-). The corpus was searched for contexts where the A- and B-terms co-occur (within \pm 9 words). It was found that in these contexts all the A-terms, including ones whose narrower A2 readings were not defined explicitly in the dictionaries (e.g. shoe), were predominantly used to contrast with their B-terms (in contexts such as shoes and boots). This suggests that the narrower $\mathrm{A} 2$ readings and the potential for $\mathrm{A} / \mathrm{B}$ contrast is well established in language for these terms. They would therefore also warrant being represented in dictionaries. This would not necessarily need to involve listing the broader and narrower readings as separate senses, as the dictionary survey demonstrated that lexicographers have at their disposal a range of definitional techniques that could be used to represent more subtle sense distinctions. Consequently different definitional techniques could be used to represent the position of a given vertical polyseme on the ambiguity-vagueness continuum.

Section 2 below provides an overview of some of the theoretical accounts of vertical polysemy, including a cognitive linguistic account where the distinction between polysemy and vagueness is viewed as being a matter of degree. The survey of the lexicographic representation of the A/B pairs is presented in section 3 and the study of the co-occurrences of the pairs in corpus data in section 4. Section 5 concludes the paper.

\section{Vertical polysemy?}

Theoretical accounts of lexical items with broader and narrower readings have tended to view the narrower readings as contextual variants of the more general readings. This means that the meaning variation has often been treated as an instance of vagueness or indeterminacy, rather than polysemy. The prioritisation of the broader reading is apparent in semantic markedness accounts of vertical polysemy, starting with Jakobson (1935 [1971], cited in Haspelmath 2006). According to the semantic markedness principle, in cases where there is only one sex-specific term for an animal species (e.g. bitch 'female dog', drake 'male duck'), the general, unmarked species term (dog, duck) can, in some contexts, be used to fill the lexical gap. ${ }^{2}$ More recent pragmatic accounts have similarly tended to view the narrowed readings as extended usages, rather than as distinct senses in their own right. For instance, Becker (2002) maintains that the A2 reading of finger (in contrast with thumb) is not a distinct sense because it can be derived inferentially from the Al reading by Grice's (1975) Maxim of Quantity. For instance,

\footnotetext{
${ }^{2}$ Kempson's (1980) lexical rule account of vertical polysemy, which is essentially a more general version of the markedness principle, similarly prioritises the broader readings of vertical polysemes. To account for the meaning variation in cases including dog/bitch, cow/bull and rectangle/square, Kempson proposes the following principle: any lexical item can be used in a narrower A2 reading that contrast with a B-term if the B-term is the only hyponym of the A-term along any one semantic dimension. However, the requirement that $\mathrm{B}$ should be the only hyponym of A is too strict. Plant, for instance, is superordinate to fern, tree, shrub, herb (among others), but plant nevertheless has a narrower A2 reading that contrasts with tree (see also Rohdenburg 1985a). Contrary to Kempson's assumption, vertical polysemy does not appear to be a type of regular polysemy, accountable for by a general lexical rule, but is rather motivated by factors such as cultural salience and prototypicality (Lehrer, 1990b).
} 
assuming that a speaker who says I've hurt my finger is making the most informative statement she can, a hearer may infer that the more specific term thumb was not used because it would have been inaccurate or untruthful in the context. Consequently, finger can be interpreted as meaning 'not thumb'.

However, semantic markedness and pragmatic accounts often fail to take into account the potential conventionalisation of the readings. Interpretations that start off as contextual usages often become semanticised (see Traugott \& Dasher 2002 and Horn 1984 , who sees the pragmatic inferences that may motivate autohyponymy as a potential mechanism of semantic change). Significantly, vertical polysemy is also not always found cross-linguistically in translational equivalents. Indeed, Becker (2002) notes that the 'thumb-excluding' A2 reading is not triggered for the German Finger. If the narrower reading were purely pragmatic, we would expect to also find it in German, to the extent that pragmatic inferences are language-independent (as is assumed at least in classical Gricean theory). But these cross-linguistic differences suggest that English and German have developed different conventions for finger and Finger, which we would expect to be encoded differently in each language's lexicon.

Another kind of argument against analysing vertical meaning variation as polysemy is presented by Lehrer (1990a). She considers the relationship between cup and mug and maintains that the fact that cup can either include mugs or exclude them does not amount to polysemy, but is rather a reflex of the prototype structure of the CUP category. That is, the flexible boundaries of CUP can either be construed more narrowly, just including prototypical cups (small vessels commonly used with a saucer), or more broadly, also including mugs as more marginal members. The same prototypical/marginal structure applies to many other A/B pairs, including shoe/boot, animal/bird, plant/tree and leather/suede. However, although the meaning variation of cup and many other A-terms is motivated by prototype category structure, their broader and narrower readings are more than just variants of a single prototype category. There is a significant difference between the broader and narrower readings in these cases, to the extent that the readings can be shown to have different truth conditions. This is a traditional ambiguity criterion, according to which an ambiguous word can be simultaneously true and false of the same referent (Quine 1960). Consequently, a word can be held to be ambiguous if it can occur in sentences of the form $p$ and not $p$ - which is shown to be the case for the broader and narrower readings of cup in (2). A1 and A2 readings can also give rise to genuine ambiguity in some contexts, as was demonstrated by (1) above.

(2) A mug is a cup [A1] but it is not a cup [A2].

The meaning variation of some A-terms can therefore look like vagueness from one perspective, and from another show symptoms of ambiguity. Such cases can be accounted for in a model of word meaning where the distinction between ambiguity and vagueness is seen as a matter of degree.

\subsection{The ambiguity-vagueness continuum and vertical polysemy}

In cognitive linguistic literature it has been proposed that ambiguity and vagueness should be treated as the end points of a continuum, rather than as dichotomous categories 
(see in particular Tuggy 1993 and Croft \& Cruse 2004). Applying this model to vertical polysemy, A2 readings that represent a prototypical subset of the A1 reading can be viewed as less than fully autonomous senses. In this respect they resemble cases such as the 'cutlery knife' and 'kitchen knife' readings of knife, or the 'text' and 'tome' readings of book, which similarly exhibit some symptoms of autonomy together with symptoms of unity (see Cruse 2000; Croft \& Cruse 2004). Such cases of meaning variation fall in between full polysemy and vagueness.

In other cases of vertical polysemy the readings are not related by prototype structure and can consequently be viewed as more clearly distinct senses - that is, closer to the 'ambiguity' end of the continuum. The 'male canine' reading of $d o g$, for instance, does not represent a prototypical subset of canines in general: male dogs are not prototypical dogs, unlike members of a specific breed such as spaniel or Alsatian. The 'male canine' reading of $d o g$ is also more clearly demarcated from the broader 'canine' reading as it adds only one additional, binary property to the intension of the broader reading, as depicted diagrammatically in (a) in Figure 1. In contrast, the boundaries demarcating a prototypical A2 reading from the A1 reading may be more ill-defined and involve multiple gradable properties (see (b) in Figure 1). For instance, cups in the narrower sense differ from cups in general in terms of their size, shape and material.
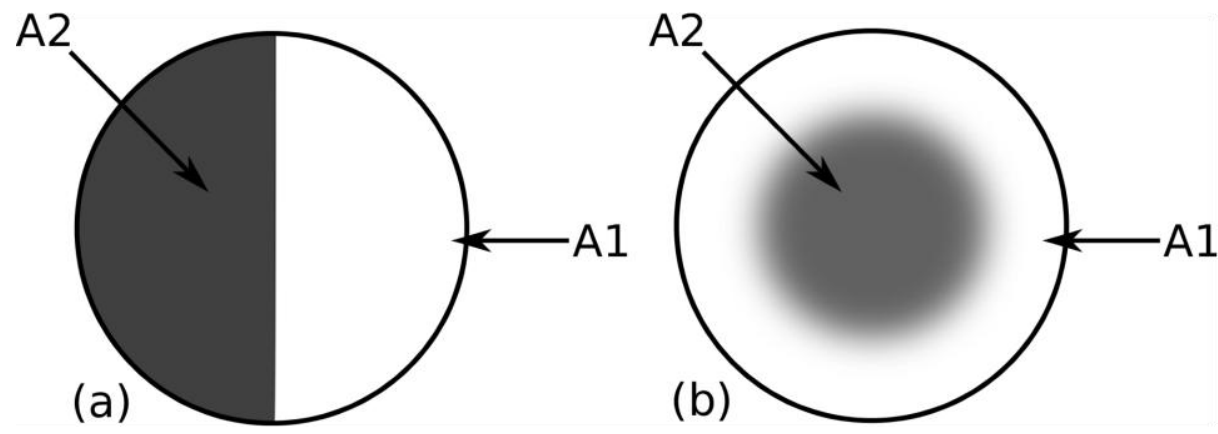

Figure 1: More and less distinct A2 readings.

In cases where the $\mathrm{A} 1$ and $\mathrm{A} 2$ readings are not fully autonomous, the distinction between them can also be more easily neutralised in certain contexts. For instance, in a compound noun such as index finger it is irrelevant whether the meaning of finger is construed more broadly or more narrowly: index finger-A 1 and index finger-A2 would both refer to the same body part. The same applies to court shoe: because court shoes always by their design only come up to the ankle, the distinction between the broader and narrower readings of shoe is neutralised as no ambiguity could ensue. In contrast, husky dog could be genuinely ambiguous between 'a canine of the Husky breed' and 'a male canine of the Husky breed' readings.

The autonomy of the A1 and A2 readings is also reflected in the range of contexts where the readings can occur. For example, cow can be used in a broader reading that includes bulls, but this reading is in fact relatively weak in that its range of uses is limited (Lyons 1977; Rohdenburg 1985a, b and Haspelmath 2006). While it is possible to refer to a group of male and female bovines collectively as cows, more explicit 
hyperonym uses are awkward (??A bull is a cow). Furthermore, cow cannot easily be

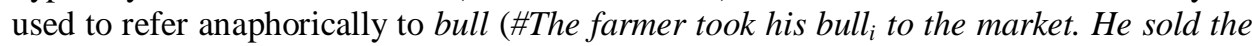
cow $_{i}$ to his neighbour). The use of weaker, less autonomous A2 readings is also more restricted. Such readings cannot co-occur with the B-term in explicitly negated contexts or other more contrastive contexts, although they can co-occur as part of an andcoordination construction (see Rohdenburg 1985a). For example, the meaning of book can be construed as excluding dictionaries, but this narrowed reading is not very strongly autonomous. Book and dictionary can co-occur in a coordination construction, but binary coordination and explicit negation are more awkward:

(3) He put all his books and dictionaries on the shelf.

(4) ?This shop sells both books and dictionaries.

(5) ?It's not a book, it's a dictionary.

To return to the point made earlier regarding the conventionalisation of the readings of a lexical item, Rohdenburg (1985a) stresses that the autonomy of the readings does not necessarily correlate with how established they are within the speech community. That is, in principle readings that are not very distinctive can nevertheless be conventionalised as distinct senses as a function of their frequency in language use (see also Langacker 1987; Bybee 2006 and other usage-based accounts of language).

This is a significant point that has implications both for theoretical accounts of polysemy, but also for the representation of word meaning in dictionaries. The aim of modern dictionaries is generally to represent the conventions of a language community, usually informed by corpus data on language use. However, dictionary definitions must also take into account the needs of the target audience (e.g. learners vs. native speakers) and practical considerations such as the size of the dictionary. Given the potential variation in distinctness and conventionality found in cases of vertical polysemy, a survey of dictionaries was carried out in order to investigate the following questions:

- How do dictionaries represent the A-terms' duality of meaning?

- On what grounds are A1 and A2 readings enumerated as distinct senses?

- (How) do dictionaries represent more subtle sense distinctions?

- How is the relationship between A- and B-terms represented (as inclusion or contrast)?

\section{Vertical polysemy in dictionaries}

To investigate the lexicographic representation of vertical polysemy, seven monolingual English dictionaries were surveyed, considering the representation of 12 pairs of A- and B-terms. 


\subsection{Dictionary survey methodology}

The $12 \mathrm{~A} / \mathrm{B}$-terms considered in the study are listed in Table 1 . The pairs were generally selected based on examples mentioned in the literature on vertical polysemy (in particular Kempson 1980; Horn 1984; Rohdenburg 1985a, b and Lehrer 1990a, b).

\begin{tabular}{|l|l|}
\hline \multicolumn{1}{|c|}{ A-term } & B-term \\
\hline dog & bitch \\
\hline cow & bull \\
\hline duck & drake \\
\hline gay & lesbian \\
\hline finger & thumb \\
\hline rectangle & square \\
\hline animal & bird \\
\hline plant & tree \\
\hline shoe & boot \\
\hline cup & mug \\
\hline coat & jacket \\
\hline leather & suede \\
\hline
\end{tabular}

Table 1: The A- and B-terms considered in the study.

The A-terms selected included four lexical items whose A2 reading is defined by a single additional binary feature (male/female), namely dog, cow, duck and gay. For all the other A-terms, the A2 reading is prototypical or otherwise less distinct definitionally.

Animal was paired with bird, although given that the narrow reading of animal denotes land-dwelling non-human mammals, the B-term could equally have been reptile, fish, insect or human (being). Animal also has an intermediate sense that excludes just humans while encompassing other members of the animal kingdom, but the focus here was on the narrowest A2 reading. Coat and jacket were considered as terms for outer garments (rather than the top part of a suit), whereby they are primarily contrasted with respect to the length of the item of clothing.

Etymologically, most A-terms' broader readings predate their narrower readings, apart from cow, whose broader reading is a non-technical use developed from the earlier 'female domesticated bovine mammal' sense (Oxford English Dictionary 2nd ed. 1989). All terms were considered primarily as nouns, with the exception of gay, whose nominal use tends to be restricted to the plural form and which was therefore also considered as an adjective. The nominal and adjectival uses of gay were generally treated under the same entry in the dictionaries and as such the discussion below does not differentiate these.

The representation of the A- and B-terms was surveyed in the following monolingual desk-size English dictionaries:

American Heritage Dictionary of the English Language. 4th edn. (2000) [AHD4]

Bloomsbury English Dictionary, New Edition (2004) [BED]

Collins English Dictionary. 9th edn. (2007) [CED9]

Oxford Dictionary of English, 2nd edn. revised (2005) [ODE2] 
Collins COBUILD Advanced Dictionary, 6th edn. (2009) [COB6]

Longman Dictionary of Contemporary English, 5th edn. (2009) [LDCE5]

Oxford Advanced Learner's Dictionary, 7th edn. (2005) [OALD7]

The first four of these dictionaries are general-purpose dictionaries aimed at native speakers (mostly by British publishers, apart from AHD4), while COB6, LDCE5 and OALD7 are monolingual learners' dictionaries. Given that vertical polysemy is not necessarily cross-linguistically constant across translational equivalents (as noted in section 2), non-native learners of English might be expected to particularly benefit from an explicit representation of the A-terms' duality of meaning.

\subsection{The representation of the A-terms}

Table 2 provides a summary of how the dictionaries represented the readings of the Aterms.

\begin{tabular}{|c|c|c|c|c|}
\hline A-term & $\mathrm{A} 1$ and $\mathrm{A} 2$ & Only A1 & $\begin{array}{c}\text { Disjunctive } \\
\text { def. }\end{array}$ & Only A2 \\
\hline $\operatorname{dog}$ & $\begin{array}{l}\text { AHD4, BED, CED9, } \\
\text { COB6, LDCE5, OALD7, } \\
\text { ODE2 }\end{array}$ & - & - & - \\
\hline cow & $\begin{array}{l}\text { AHD4, BED, CED9, } \\
\text { LDCE5, ODE2 }\end{array}$ & OALD7 & - & COB6 \\
\hline duck & $\begin{array}{l}\text { AHD4, BED, CED9, } \\
\text { LDCE5, OALD7, ODE2 }\end{array}$ & COB6 & - & - \\
\hline gay & AHD4 & $\begin{array}{l}\text { BED, CED9, COB6, } \\
\text { LDCE5, OALD7, ODE2 }\end{array}$ & - & - \\
\hline finger & - & AHD3, BED, CED9 & $\begin{array}{l}\text { OALD7, } \\
\text { ODE2 }\end{array}$ & $\begin{array}{l}\text { COB6, } \\
\text { LDCE5 }\end{array}$ \\
\hline rectangle & - & $\begin{array}{l}\text { AHD4, BED, CED9, } \\
\text { COB6, LDCE5, ODE2 }\end{array}$ & - & OALD7 \\
\hline animal & $\begin{array}{l}\text { AHD4, BED, CED9, } \\
\text { COB6, LDCE5, OALD7, } \\
\text { ODE2 }\end{array}$ & - & - & - \\
\hline plant & $\begin{array}{l}\text { AHD4, BED, } \\
\text { ODE2 }\end{array}$ & COB6, LDCE5, OALD7 & - & - \\
\hline shoe & - & $\begin{array}{l}\text { AHD4, BED, CED9, } \\
\text { COB6, LDCE5, OALD7, } \\
\text { ODE2 }\end{array}$ & - & - \\
\hline
\end{tabular}




\begin{tabular}{|l|l|l|l|l|}
\hline \multicolumn{1}{|c|}{ A-term } & \multicolumn{1}{|c|}{ A1 and A2 } & \multicolumn{1}{|c|}{ Only A1 } & \multicolumn{1}{c|}{$\begin{array}{c}\text { Disjunctive } \\
\text { def. }\end{array}$} & Only A2 \\
\hline \multirow{2}{*}{ cup } & - & $\begin{array}{l}\text { AHD4, BED, CED9, } \\
\text { COB6, LDCE5, OALD7, } \\
\text { ODE2 - }\end{array}$ & - \\
\hline \multirow{2}{*}{ coat } & - & $\begin{array}{l}\text { AHD4, BED, CED9, } \\
\text { COB6, LDCE5, OALD7, } \\
\text { ODE2 - }\end{array}$ & - \\
\hline \multirow{2}{*}{ leather } & - & $\begin{array}{l}\text { AHD4, BED, CED9, } \\
\text { LDCE5, COB6, OALD7, } \\
\text { ODE2 - }\end{array}$ & - \\
\hline
\end{tabular}

Table 2: The representation of the A-terms in the dictionaries

Just over a third (36\%) of all the A-term definitions (30 of the total 84 A-term entries in all the dictionaries combined) involved some form of polysemous representation. The A1 and A2 readings were either listed as separate senses of equal status or one was shown as a subsense (ODE2 particularly made use of subsenses to highlight the relationship between the readings). ${ }^{3}$ As Table 2 shows, all the dictionaries represented $d o g$ as vertically polysemous, and most also did so for duck and cow. Animal was also represented as a vertical polyseme in all the dictionaries surveyed, with some dictionaries enumerating three vertically related readings, including the intermediate 'non-human animate being' reading.

However, as Table 2 shows, the majority of the A-term entries did not enumerate the A1 and A2 readings explicitly, usually only listing the broader reading (although notably only the narrower reading was given for finger in COB6 and LDCE5 and for rectangle in $O A L D 7)$. However, even though many of the entries did not separate the A2 reading as a distinct sense, they made use of other definitional techniques that served to indicate the duality of the A-term's meaning in relation to the B-term. Notably, in two of the dictionaries finger was given a disjunctive definition that covers both the A1 and the A2 readings as alternatives (see (6), emphasis added). However, traditional defining principles disfavour disjunctions (Geeraerts 2001), perhaps explaining why this type of definition was not used more commonly.

(6) finger $[\ldots]$ one of the four long thin parts that stick out from the hand (or five, if the thumb is included) (OALD7)

\footnotetext{
${ }^{3}$ Among the polysemous representations, the broader A1 reading was generally listed above the narrower A2 reading. The main exception to this was cow, whose the narrower 'female bovine' reading was given first in all the dictionaries that distinguished its two senses. The learners' dictionaries also placed the narrower sense(s) of animal before the more general one, whereas all the dictionaries aimed at native speakers listed the broadest sense first. It is not entirely clear why the ordering of the senses of animal was different in the two types of dictionaries given that their policies on sense ordering were not consistently different. Most of the general-purpose and learners' dictionaries stated that senses were ordered on the basis of their frequency in language use.
} 
Information regarding the A-terms' duality of meaning was also sometimes included in usage notes. Three of the dictionaries ( $A H D 4, B E D$ and $O D E 2)$ included a separate usage note for gay, which, among other things, mentioned the existence of the broader and narrower readings (see (7)). Only AHD4 also enumerated the A1 and A2 readings separately, however.

(7) Gay in its modern sense typically refers to men (lesbian being the standard term for homosexual women) but in some contexts it can be used of both men and women. (ODE2)

In addition to such separate usage notes, the definition text for some of the other lexical items also included a metalinguistic comment on usage, through the use of adverbs such as sometimes or often:

(8) cow [...] A cow is a large female animal that is kept on farms for its milk. People sometimes refer to male and female animals of this species as cows. (COBO)

(9) finger $[\ldots]$ any of the digits of the hand, often excluding the thumb. (CED9)

The definition in (8) particularly makes an explicit comment on usage, but the use of often in (9) may also be interpreted as describing the use of the definiendum rather than characteristics of the referent.

Specifying adverbs such as typically, usually and especially also occurred in many of the definitions. As Geeraerts (2001) notes, such adverbs are commonly used to include extensional or encyclopaedic elements in dictionary definitions, and often serve the purpose of characterising prototypical category members. As discussed above, in many vertical polysemes the A2 reading designates a prototypical subset of the broader A1 category. In reflection of this, the definition of the A1 reading often (in 17 out of the 50 A-term entries that only listed the A1 reading) implicitly characterised the A2 reading through the use of such specifying adverbs (see examples (10)-(12)).

(10) coat $[\ldots]$ An outer garment with sleeves, worn outdoors and typically extending below the hips. (ODE2)

(11) shoe [...] A durable covering for the human foot, made of leather or similar material with a rigid sole and heel, usually extending no higher than the ankle. (AHD4)

(12) rectangle $[\ldots]$ a two-dimensional geometric figure formed of four sides in which each angle is a right angle, especially one with adjacent sides of different length $(B E D)$

Of course, many dictionary definitions generally include information about prototypical referents - for instance, the $O D E 2$ defines tree as 'a woody perennial plant, typically having a single stem or trunk'. Such information therefore does not necessarily mean that the prototypical subset constitutes a (semi-)autonomous narrower reading. Including information about prototypical referents within the A-term's definition is only equivalent to Lehrer's (1990a) analysis of the A1 and A2 readings as variants of a single prototype category. However, the A2 reading can be demarcated more clearly if the prototypical referents are explicitly contrasted with the B-term, as in (13) and (14). 
(13) rectangle [...] a plane figure with four straight sides and four right angles, especially one with unequal adjacent sides, in contrast to a square. (ODE2)

(14) plant $[\ldots]$ a living thing that has leaves and roots and grows in earth, especially one that is smaller than a tree. (LDCE5)

Making an explicit reference to the contrast relationship between the A- and B-terms was one way in which the $\mathrm{A} / \mathrm{B}$ relationship was represented in the dictionaries.

\subsection{The representation of the $A / B$ relationship}

Many of the A-term entries in fact referred explicitly to the potential contrast with the Bterm. Overall, 31 of the 84 A-term entries surveyed included some reference to the contrast with the B-term - see Table 3 .

\begin{tabular}{|l|l|}
\hline \multicolumn{1}{|c|}{ A-term } & Dictionaries explicitly mentioning the A/B contrast \\
\hline dog & LDCE5, OALD7, COB6 \\
\hline cow & LDCE5, OALD7 \\
\hline duck & CED9, LDCE5, OALD7, ODE2 \\
\hline gay & AHD4, BED, LDCE5, OALD7, ODE2 \\
\hline finger & AHD4, BED, CED9, LDCE5, OALD7, ODE2 \\
\hline rectangle & LDCE5, ODE2 \\
\hline animal & COB6, LDCE5, OALD7, ODE2 \\
\hline plant & BED, CED9, LDCE5, OALD7, ODE2 \\
\hline shoe & - \\
\hline cup & - \\
\hline coat & - \\
\hline leather & - \\
\hline
\end{tabular}

Table 3: A-terms defined in explicit contrast with the B-term. In the dictionaries marked in bold only the $\mathrm{A} 1$ reading was given for the A-term.

17 of the 31 entries mentioning the $\mathrm{A} / \mathrm{B}$ contrast defined the $\mathrm{A} 2$ reading as a separate sense, and the reference to the contrast was included with that definition. However, the other 14 entries gave only the A1 reading, and the potential for contrast with the B-term was noted as part of the definition text, as in (13) and (14) above. The A/B contrast was usually indicated by defining the A2 reading negatively in opposition to the B-term. This often provides the simplest way of defining the A2 reading: finger-A2 is one which is not the thumb, and animal-A2 is one which is not a human, bird, reptile or fish. In contrast, in cases where the A2 reading can be defined with the addition of a single feature (e.g., 'male dog', 'female duck'), the A/B contrast was more commonly indicated through cross-references (e.g. duck... compare DRAKE), examples (e.g. Is this a dog or a bitch?) or usage notes (as in some of the entries for gay).

As Table 3 shows, the potential A/B contrast was indicated more frequently for some terms than others. Notably, almost all the dictionaries (with the exception of COB6) mentioned the contrast between finger and thumb. Plant was also frequently contrasted 
with tree, as was gay with lesbian. The narrower reading of animal was also almost always defined in opposition to bird, and usually also in opposition to human (being), reptile and fish. On the other hand, none of the dictionaries noted explicitly the potential contrast for shoe/boot, cup/mug, coat/jacket and leather/suede.

The A/B contrast relationship was generally only indicated explicitly in the A-term entries, although a handful of the B-term entries included a cross-reference to the A-term (e.g. the LDCE5 entry for square cross-referred to rectangle). More commonly, the Bterm definition texts used the A-term as the genus term, that is, defined B as a type of A (e.g. mug: a large cup). In keeping with the classical genus-differentiae defining style, this implies that $\mathrm{B}$ is a direct hyponym of $\mathrm{A}$. The majority of the B-term definitions $(52 \%)$ used the A-term as the genus term. For instance, all the dictionaries defined drake as a type of duck, tree as a type of plant and suede as a type of leather. In cases such as $d u c k$, where the A-term is defined explicitly as a vertical polyseme, the genus term must of course be interpreted in the broader A1 reading. Consequently this representation prioritises the inclusion (rather than contrast) relationship between the A- and B-terms.

On the other hand, many of the B-term definitions (20\%) used the same genus term as the A-term and thus, in principle, represented the two as co-hyponyms or sister terms. ${ }^{4}$ In a few instances this was consistent with the representation of the A-term. As noted above, COB6 and LDCE5 only included the A2 reading of finger - and both also defined finger and thumb with the same genus term, part (of your hand). However, in most cases only the A1 reading was given for the A-term but the B-term was not defined as a type of $\mathrm{A}$, but rather both $\mathrm{A}$ and $\mathrm{B}$ were defined as subordinates of the same genus term. For example, although most of the dictionaries defined rectangle only in the broader A1 reading, they did not define square as a type of rectangle, but instead used the more general figure or shape as the genus term for both rectangle and square. On the basis of such definitions, it is not clear whether the relationship between rectangle and square should be interpreted as one of inclusion or contrast. Such ambiguities could be taken to reflect the dual relationship that holds between the A- and B-terms - but they may simply be accidental inconsistencies introduced by different lexicographers. It is in fact well known that dictionaries' representation of taxonomic relations through genus term choice is often imperfect and prone to ambiguities and inconsistencies (see Ide \& Véronis 1993 for discussion). Consequently it is difficult to interpret what the genus terms used in the definitions tell us about the relationship between the A- and B-terms.

\subsection{A- and B-terms in dictionaries: a summary}

Overall, the survey shows that dictionaries have at their disposal a range of methods for representing the A-terms' duality of meaning in relation to the B-term - from explicitly enumerating the $\mathrm{A} 1$ and $\mathrm{A} 2$ readings to including information about the $\mathrm{A} 2$ reading and the A/B-term contrast within the definition of the A1 sense. Applied consistently, such

\footnotetext{
${ }^{4}$ The remaining $28 \%$ of the B-term entries involved a mixture of definition types, including cases where the genus term of B was a hyponym of the genus term of A (e.g. AHD4 defines shoe as a type of covering, but boot as a type of footgear) and cases where the taxonomic relationship was unclear (e.g. ODE2 defined gay as 'a homosexual' and lesbian as 'a homosexual woman').
} 
methods could potentially be used to represent the gradable nature of sense distinctions, in keeping with the ambiguity/vagueness continuum model of polysemy.

It was notable that cases where the A1 and A2 readings were enumerated separately were generally those where the narrower reading is a sex-specific subclass of the broader one (in particular cow, $d o g$ and $d u c k$ ). Representing these A-terms as vertical polysemes can be justified given that their A1 and A2 readings can be viewed as being more distinctive - compared to cases where the A2 reading represents a prototypical subset of the $\mathrm{A} 1$ reading.

However, some of the less distinctive A2 readings were also frequently enumerated separately. Although the A2 readings of animal and plant are prototypical, most of the dictionaries represented these lexical items explicitly as vertical polysemes. Notably some of the learners' dictionaries also only gave the narrower readings for finger and rectangle and many of the other dictionaries at least noted the potential for contrast between finger and thumb and rectangle and square. In comparison, all the dictionaries defined shoe, cup, coat and leather only in the broader sense, and none of them explicitly mentioned their potential for contrast with the B-terms.

The question, then, is why the duality of meaning and potential for $\mathrm{A} / \mathrm{B}$ contrast should be represented explicitly for some terms but not others. One possibility is that the narrower readings of shoe, cup, coat and leather are less conventionalised or they have a weaker potential to contrast with their B-terms compared to animal, finger and other terms whose narrower readings and potential for $\mathrm{A} / \mathrm{B}$ contrast were represented in the dictionaries. This hypothesis was explored by analysing data on the use of the A/B pairs in natural language.

\section{A- and B-term co-occurrence in language use}

Given that the vertical meaning variation of an A-term reflects the semantic relationship it contracts with the B-term (inclusion or contrast), we can gain some insight into the occurrence of the $\mathrm{A} 1$ and $\mathrm{A} 2$ readings in language by examining contexts where the Aand B-terms co-occur. Although this takes into account only a subset of the contexts where the A-term can be used, the A-terms examined here generally have a significant collocative relationship with their B-terms. Therefore, the A/B co-occurrence contexts represent an important subset of the A-terms' usage contexts. A commonly used measure of collocation strength is the Mutual Information score, which compares the probability of the co-occurrences of the terms with the probability of their occurring independently of each other (Church \& Hanks 1990). In general, an MI score higher than 3 can be thought to indicate a significant collocation (Church \& Hanks ibid.). The average A/B pair MI score was 5.98 - the highest scoring pair was gay/lesbian (10.25) and the lowest plant/tree (2.61). Plant/tree was also the only A/B pair whose MI score fell below the threshold value of 3 . Although this means that plant/tree do not have a very strong relationship, we can nevertheless be confident that there is an association between them. This is based on another measure often used to find significant collocations, the t-score. T-score is a measure of the confidence with which we can assume that there is an association between the collocates. The critical value for assuming this with a $95 \%$ 
confidence is 2.576 (Manning \& Schütze 1999), and the t-scores of all the A/B pairs were above this threshold, including plant/tree (t-score of 14.995).

Although the A/B pairs thus form significant collocations, it is of course not necessarily the case that the frequencies of the $\mathrm{A} 1$ and $\mathrm{A} 2$ readings within the contexts where the A- and B-terms co-occur are representative of the global frequencies of these readings. Nevertheless, this methodology provides relevant information on how the relationship between the A- and B-terms is realised in language; to what extent they occur as a hyperonym/hyponym pair or as contrasting, incompatible terms. It is also informative to compare the different A/B pairs, to examine whether those terms whose potential for contrast is represented in the dictionaries are more likely to be used in contrastive ways. For the purposes of data coding, the explicit presence of the B-term also helped disambiguate the intended reading of the A-term, which can otherwise be unclear (given that the distinction between the A1 and A2 readings is often very subtle or easily neutralised). Limiting the focus to contexts where the A- and B-term co-occur was also useful for eliminating noise from contexts where the terms are used in other senses not relevant to the investigation.

\subsection{Corpus study methodology}

The British National Corpus (Davies 2004-) was searched for collocations of the A- and B-terms within a span of \pm 9 words. This span was the largest permitted by the Brigham Young University online corpus interface and was selected in order to include as many co-occurrence contexts as possible. The searches were limited to nominal uses, except in the case of gay/lesbian, where the part of speech of the search terms was left unspecified in order to collect both nominal and adjectival uses.

First any contexts where either the A- or the B-term occurred in an irrelevant sense were set aside ( 71 of the total 1693 contexts or $4.2 \%$ ). These included proper noun uses (e.g. the surname Boot) or uses involving other senses of the words (e.g. plant in the 'industrial machinery' sense or bitch as an insult). ${ }^{5}$ Metalinguistic uses of the terms were also discounted (e.g. knee-jerk reactions to the very mention of the words lesbian and gay). Any duplicated contexts and cases where the A-term co-occurred with two instances of the B-term within the 9-word span (e.g. Do you want your chocolate in a cup or in a mug? A mug is bigger) were counted only once. Such duplicates numbered 36 contexts, $2.8 \%$ of the total search results.

The remaining 1586 co-occurrence contexts were then coded for the meaning expressed by the A-term, specifically whether it was used in the broader A1 reading or in the narrower $\mathrm{A} 2$ reading in relation to the B-term. Contexts that were coded as involving the $\mathrm{A} 1$ reading included those containing constructions that are known to typically house hyperonym-hyponym pairs (Hearst 1992; Mititelu 2006). These included Bs are As; Bs and other As; As, including Bs; such As as Bs and As, especially Bs. Contexts where the A-term referred anaphorically to the B-term antecedent (e.g. (15)) and contexts where

\footnotetext{
${ }^{5}$ A large proportion (33.3\%) of the uses of coat and jacket were excluded because the terms were used to refer to the top part of a suit rather than to an outer garment.
} 
the B-term followed the A-term with the function of exemplification (as in (16)) were also coded as involving the A1 reading. ${ }^{6}$

(15) The best-developed colour vision of any creatures, though, is that of birds. These animals not only have five pigments in their retinas...

(16) I've got a big wardrobe at the moment but it mainly consists of shoes -- I bought these boots from Red or Dead.

Contexts where the A-term was modified with an adjective to contrast with B (e.g. male dog) were also coded as A1, as were cases where the A-term was used with generic reference as the attributive noun in a compound (as in a shoe shop with some wellington boots outside) or, in the case of gay, as an adjectival modifier (a gay man). Some of the animal terms also occurred in the A1 reading as the name of a species (e.g. Tufted Duck).

Contexts that were coded as involving the narrower A2 reading included cases where the A- and B-terms occurred within a construction that is associated with lexical contrast. Work on antonymy (esp. Mettinger 1994; Jones 2002; Davies 2012) has identified lexico-grammatical constructions with distinct discourse functions that are associated with the co-occurrence of opposing meanings. Importantly, these constructions are themselves associated with contrastive semantics, which means that they may induce a contrast relation between meanings that are not normally - or not always - construed as opposites (Murphy 2003; Davies 2012). ${ }^{7}$ These structures include coordination constructions (both simple $A$ and $B$ coordination and binary coordination, as in both $A s$ and $B s)$, negated structures ( $A$, not $B$; As instead of $B s$ etc.) and contexts expressing comparison (e.g. bitches tend to live longer than dogs) or transition (e.g. shoes (loafers and royals) were replacing boots). ${ }^{8}$ The A- and B- terms were similarly used in contrastive ways in structures involving lexico-grammatical parallelism (see Davies 2012) - as illustrated by (17).

\footnotetext{
${ }^{6}$ As discussed in 2.1, only very strong A1 readings can be used to refer anaphorically to the Bterm. Almost all the A-terms occurred in anaphoric contexts at least once in the data, with the exception of cow, coat and leather. For cow, this was to be expected (see the discussion above), whereas the non-occurrence of coat/jacket and leather/suede in anaphoric contexts is likely to be due to the low number of co-occurrence contexts that were analysed for these terms, rather than a reflection of the weakness of their A1 readings.

${ }^{7}$ The A/B pairs, when they occur in these constructions, are not necessarily opposites, that is, in a binary contrastive relationship. Although in some cases the A/B contrast is binary (esp. in cases where male and female individuals are contrasted), in other cases the A2 reading and the B-term are instead in a relationship of co-hyponymy, involving a contrast between multiple categories at the same taxonomic level. This is made explicit when A and B occur in multiple coordination constructions, as in plants, shrubs and trees.

${ }^{8}$ Simple and-coordination structures were the most common context within the data, making up $56 \%$ of all the coded contexts (808/1438 contexts) and $70 \%$ of the contexts coded as involving the A2 reading (808/1149 A2 contexts). As discussed above in 2.1, and-coordination constructions are indicative of only a weak contrastive relationship. However, almost all the A/B pairs also occurred at least once in a more strongly contrastive context (such as a binary coordination construction or a negated structure). The only exceptions were coat/jacket and leather/suede, but the non-occurrence of such stronger contrastive contexts for these pairs may be due to scarcity of data.
} 
(17) Without water, nothing can live. Trees die, plants die, animals die, people die

Contexts where A and B were used in contrastive semantic roles were also coded as involving the A2 reading. These mainly involved the use of terms for male and female animals in contexts relating to mating or breeding (e.g. All the drakes are chasing the duck).

Any contexts where there was any uncertainty as to the intended meaning of the Aterm were set aside and discounted from the calculations (94 [5.9\%] out of the 1586 contexts). Similarly discounted were any cases where the A-term was modified in such a way that the distinction between the A1 and A2 readings was neutralised, as in the case of index finger and court shoes discussed in 2.1 above (54 contexts or $3.4 \%$ ).

\subsection{A1 and A2 readings in co-occurrence contexts}

Table 4 shows the frequencies of the readings of the A-terms in the corpus data.

\begin{tabular}{|l|l|l|l|l|l|}
\hline A-term & A1 & A1 \% & A2 & A2 \% & Total \\
\hline cow & 1 & 1.1 & 91 & 98.9 & 92 \\
\hline finger & 5 & 3.2 & 149 & 96.8 & 154 \\
\hline leather & 1 & 6.7 & 14 & 93.3 & 15 \\
\hline rectangle & 2 & 6.9 & 27 & 93.1 & 29 \\
\hline cup & 1 & 8.3 & 11 & 91.7 & 12 \\
\hline coat & 2 & 9.5 & 19 & 90.5 & 21 \\
\hline shoe & 11 & 10.5 & 94 & 89.5 & 105 \\
\hline gay & 95 & 20.4 & 370 & 79.6 & 465 \\
\hline animal & 67 & 24.0 & 212 & 76.0 & 279 \\
\hline plant & 57 & 37.0 & 97 & 63.0 & 154 \\
\hline dog & 22 & 40.0 & 33 & 60.0 & 55 \\
\hline duck & 25 & 43.9 & 32 & 56.1 & 57 \\
\hline
\end{tabular}

Table 4: Raw figures and percentage proportions of the A1 and A2 readings of the A-terms in the corpus data, ranked according to the frequency of the $\mathrm{A} 2$ reading.

It is striking that for all $12 \mathrm{~A}$-terms, the majority of the co-occurrence contexts involved the narrower A2 reading. On average the A/B pairs were used in contrastive ways in $82.4 \%$ of the contexts, or 4.7 times as often as they were used as a hyperonym/hyponym pair. Given that the contexts where the A- and B-terms co-occur represent only a subset of the contexts where the A-term is used, the frequencies in Table 4 should naturally not be interpreted as reflecting the global frequencies of the broader and narrower readings of the A-terms. For example, just on the basis of introspection, we may assume that dog is more likely to generally be used to refer to the species rather than to male animals. Nevertheless, the high frequencies of the A2 readings in the data show that all the A/B pairs have a strong potential to be used in contrastive ways in contexts where they cooccur. Thus we can infer that their narrower readings are relatively conventionalised. In 
comparison, consider the pair book and dictionary. Within 68 book/dictionary cooccurrence contexts retrieved from the BNC (following the same procedure as for the A/B-terms), book was used as a co-hyponym of dictionary only $7.4 \%$ of the time $(5 / 68$ contexts). This suggests that the A2 reading of book is not as strongly established as the A2 readings of the 12 A-terms considered here.

There are a number of factors that may have contributed to the high frequency of the A2 readings in the A/B co-occurrence contexts. The very high frequency at which cow was used in the A2 reading in contrast with bull was unsurprising, given that the A1 reading of cow is generally held to be a weaker usage. But in other cases the explicit presence of the B-term may have made the potential A/B contrast relationship particularly salient. In the case of dog/bitch and duck/drake, for example, the B-term tends to occur in contexts concerning animal husbandry or biology. In such contexts the distinction between male and female animals is particularly relevant, and thus the Aterm would be more likely to occur in the sex-specific narrower reading. A similar argument could also apply to some of the other A/B pairs. It is also worth noting that although the \pm 9 word span that was used in the collocate search was the largest allowed by the corpus tool, some of the relevant co-occurrence contexts would still have been excluded. In particular, anaphoric reference using the superordinate term can occur over long distances (possibly even across paragraphs - see Ariel 1988), and thus many such uses of the A1 reading were inevitably left out of consideration.

\subsection{Comparing different A-terms}

While all the A-terms occurred more frequently in the narrower A2 reading in the A/B co-occurrence contexts, Table 4 shows that some A/B pairs were used in contrastive ways more frequently than others. Comparing the frequencies of the A2 readings of different A-terms suggests that A-terms whose narrower A2 readings were explicitly represented in the dictionaries do not necessarily have the strongest potential to contrast with their B-terms.

Overall, a chi-square test shows that highly significant differences exist between the frequencies of the A1 and A2 readings of the different A-terms $\left(X^{2}=125.06, d f=11\right.$, $\mathrm{p}<0.001)$. Pairwise comparisons were then performed between all the different A-terms by using the Marascuilo procedure to identify statistically significant differences (see Table 5).

\begin{tabular}{|l|l|l|l|}
\hline Pairwise comparison & Difference & \multicolumn{1}{|c|}{$\boldsymbol{\alpha}$} & Decision \\
\hline cow vs. animal & -0.2293 & $<0.0001$ & $\mathrm{~S}$ \\
\hline cow vs. duck & -0.4277 & $<0.0001$ & $\mathrm{~S}$ \\
\hline cow vs. plant & -0.3593 & $<0.0001$ & $\mathrm{~S}$ \\
\hline cow vs. gay & -0.1934 & $<0.0001$ & $\mathrm{~S}$ \\
\hline cow vs. dog & -0.3891 & 0.0004 & $\mathrm{~S}$ \\
\hline finger vs. animal & -0.2077 & $<0.0001$ & $\mathrm{~S}$ \\
\hline finger vs. gay & -0.1718 & $<0.0001$ & $\mathrm{~S}$ \\
\hline finger vs. plant & -0.3377 & $<0.0001$ & $\mathrm{~S}$ \\
\hline
\end{tabular}




\begin{tabular}{|l|l|c|l|}
\hline Pairwise comparison & Difference & $\boldsymbol{\alpha}$ & Decision \\
\hline finger vs. duck & -0.4061 & 0.0001 & $\mathrm{~S}$ \\
\hline finger vs. dog & -0.3675 & 0.0018 & $\mathrm{~S}$ \\
\hline shoe vs. plant & -0.2654 & 0.0021 & $\mathrm{~S}$ \\
\hline shoe vs. duck & -0.3338 & 0.0297 & $\mathrm{~S}$ \\
\hline shoe vs. dog & -0.2952 & 0.1209 & $\mathrm{AS}$ \\
\hline rectangle vs. plant & -0.3012 & 0.0114 & $\mathrm{~S}$ \\
\hline rectangle vs. duck & -0.3696 & 0.0343 & $\mathrm{~S}$ \\
\hline rectangle vs. dog & -0.3310 & 0.1184 & $\mathrm{AS}$ \\
\hline
\end{tabular}

Table 5: The output of the Marascuilo procedure showing significant differences between different A-terms (S) and differences approaching significance (AS). All other pairwise comparisons were non-significant.

As expected, cow was significantly more likely to occur in the A2 reading than many of the other A-terms. However, finger also occurred almost always (in 96.8\% of the contexts considered) in the narrower A2 reading, significantly more often than many of the other A-terms. Given that finger and thumb thus have a high potential to contrast, we can infer that the narrower reading of finger is very conventionalised. We might therefore expect it to be represented in dictionaries (and in the mental lexicons of language users - contra Becker 2002). A few of the dictionaries did define the A2 reading of finger explicitly, but not all of them did so. Most of the dictionaries did, however, note the potential contrast between finger and thumb.

In contrast, none of the dictionaries defined shoe in the narrower A2 reading or included explicit information regarding the potential contrast between shoe and boot. Shoe and boot have a fairly strong collocative relationship (MI score of 5.29 and t-score of 11.075) and the data in Table 4 shows that shoe and boot are very likely to contrast in contexts where they co-occur. In this respect shoe in fact differed significantly (or almost significantly) from duck, dog and plant, all of which occurred in the narrower reading less often in the data. ${ }^{9}$ It is not clear why the dictionaries were reluctant to represent the duality of the meaning of shoe and its potential contrast with boot. It may be a reflection of the fact that the readings of shoe are related by prototype structure and consequently less distinctive. However, prototypical and less distinctive A2 readings should also have their place in dictionaries in cases where they are conventionalised. As the data here suggests that the potential contrast between shoe and boot is well established, it would also warrant some recognition in the lexicographic representation of the word's meaning.

Although all the A-terms were more frequently used in the narrower A2 reading in the data, duck and dog were the least likely to occur in the narrower reading compared to

${ }^{9}$ It is worth noting that although leather, cup and coat also occurred more frequently in the narrower reading than many of the other A-terms (see Table 4) so few co-occurrence contexts were analysed for these terms that they did not differ statistically from any of the other A-terms. Thus although the data does suggest that leather, cup and coat are frequently used in the narrower reading in contexts where they co-occur with their B-terms, given the scarcity of data, we must be careful when drawing conclusions regarding the relative conventionality of their A2 readings. 
the other A-terms - and more likely to occur in the broader reading. In this they differed significantly from some of the other A-terms. A possible explanation for the comparatively higher frequencies of the A1 readings of $d u c k$ and $d o g$ in the data is that their broader A1 readings are more established than their A2 readings. As sex-specific terms for animals, the narrower A2 readings of duck and $d o g$ can be viewed as semitechnical usages, restricted to specialised registers - unlike their broader readings. This might explain why the broader, hyperonym uses of $d u c k$ and $d o g$ were relatively more dominant in the data. That almost all the dictionaries listed the A2 readings of $d o g$ and duck as separate senses may therefore reflect the definitional distinctiveness of these readings rather than how strongly established they are.

\section{Discussion and conclusion}

Reflecting on the lexicographic representation of vertical polysemes, Rohdenburg (1985b: 71) remarks that "lexicographers tend to concentrate on the general sense of the unmarked term [i.e., the A-term]" and "on the whole, the specific sense of the unmarked term is sadly neglected by both lexicographers and general linguists". The survey of seven monolingual English dictionaries suggests that the specific senses that tend to be 'neglected' are those that are relatively less distinctive, generally representing prototypical subsets of the more general reading of the A-term. This is despite the fact that these narrower readings are established in language use, insofar as they occur very frequently in contexts where the A-terms co-occur with the B-terms. Cases where the vertical polysemy of the lexical item was represented more explicitly in the dictionaries tended to involve A1 and A2 readings that were more distinctive and not related by prototype structure. This is justifiable given that such cases would fall closer to the 'ambiguity' end of the ambiguity-vagueness continuum. However, the corpus data, particularly in the case of $d o g$ and $d u c k$, shows that A-terms whose narrower readings are more distinctive definitionally do not always have the strongest potential to contrast with their B-terms, and their narrower readings are not necessarily as strongly established than those of other vertical polysemes.

Some of the less distinctive narrower readings were also defined as separate senses (especially for animal, plant and to a lesser extent finger and rectangle), but the corpus data suggests that there would be equally strong grounds for dictionaries to also represent the duality of meaning of terms such as shoe. This would not necessarily have to involve listing the broader and narrower readings as separate senses, as the dictionary survey showed that lexicographers have at their disposal a range of more subtle methods for representing meaning variation. This includes disjunctive definitions, metalinguistic comments on usage and the inclusion of information about prototypical category members and the potential for A/B contrast within the definition text. Such definitional techniques could be used for cases where the A1 and A2 readings are less distinctive but nevertheless conventionalised. Used systematically, different definitional techniques could potentially be used to reflect the gradable distinctness of sense, in keeping with the model of polysemy suggested in cognitive linguistic literature.

Comparing the general purpose and learners' dictionaries, it was notable that in a few cases the learners' dictionaries were more prepared to represent the narrower readings of 
the A-terms as distinct senses. For instance, two of the learners' dictionaries defined finger only in the narrower reading, while many of the general-purpose dictionaries prioritised the broader reading, even though the corpus data suggests that the narrower reading of finger is highly established. The learners' dictionaries' prioritisation of the narrower readings may reflect the known cross-linguistic differences in the meaning of 'finger', which are important to bring to the attention of learners of English.

The corpus data showed that in contexts where they co-occur, the A- and B-terms are more frequently used in contrastive ways than as a hyperonym/hyponym pair. This is an interesting finding with relevance for the general function of lexical contrast in language. Research on opposites has shown that words with opposing meanings tend to co-occur significantly more frequently than would be expected by chance (e.g. Justeson \& Katz 1991; Jones 2002). The pattern demonstrated here is the reverse: when words that have an established potential to either contrast or function as a hyperonym/hyponym pair occur in the same context, they tend to be used in contrastive ways. This pattern warrants further investigation, as does a more fine-grained analysis of the types of lexicogrammatical contexts where vertical polysemes can occur, as evidence of the relative strength of their broader and narrower readings.

\section{References}

\section{Dictionaries}

American Heritage Dictionary of the English Language, $4^{\text {th }}$ edn. (2000) Boston: Houghton Mifflin.

Bloomsbury English Dictionary, New Edition (2004) London: Bloomsbury.

Collins English Dictionary, $9^{\text {th }}$ edn. (2007) Glasgow: HarperCollins

Oxford Dictionary of English, $2^{\text {nd }}$ edn. revised (2005) Oxford: Oxford University Press.

Collins COBUILD Advanced Dictionary, $6^{\text {th }}$ edn. (2009) London: HarperCollins.

Longman Dictionary of Contemporary English, $5^{\text {th }}$ edn. (2009). Harlow: Longman Pearson.

Oxford Advanced Learner's Dictionary, $7^{\text {th }}$ edn. (2005) Oxford: Oxford University Press.

\section{Other sources}

Ariel, Mira (1988). "Referring and accessibility". Journal of Linguistics, 24(1), 65-87. DOI: $10.1017 /$ S0022226700011567

Becker, Thomas (2002). "Autohyponymy: Implicature in lexical semantics, word formation, and grammar". Journal of Germanic Linguistics, 14(2), 105-136. DOI: $10.1017 /$ S 1470542702000065

Bybee, Joan (2006). "From usage to grammar: the mind's response to repetition". Language, 82(4), 711-733. 
Church, Kenneth \& Patrick Hanks (1990). "Word association norms, mutual information, and lexicography". Computational Linguistics, 16(1), 22-29.

Croft, William \& D. Alan Cruse (2004). Cognitive linguistics. Cambridge: Cambridge University Press.

Cruse, D. Alan (2000). "Aspects of the microstructure of word meanings". In Y. Ravin \& C. Leacock (Eds.), Polysemy: Theoretical and computational approaches (30-51). Oxford: Oxford University Press.

Davies, Matt (2012). "A new approach to oppositions in discourse: The role of syntactic frames in the triggering of non-canonical oppositions". Journal of English Linguistics, 40(1), 47-73.

Davies, Mark (2004-). BYU-BNC. (Based on the British National Corpus from Oxford University Press). Available online at http://corpus.byu.edu/bnc/. DOI: 10.1177/0075424210385206

Geeraerts, Dirk (1993). "Vagueness's puzzles, polysemy’s vagaries". Cognitive Linguistics, 4(3), 223-272. DOI: 10.1515/cogl.1993.4.3.223

Geeraerts, Dirk (2001). "The definitional practice of dictionaries and the cognitive conception of polysemy". Lexicographica, 17, 6-21. DOI: 10.1515/9783110244212.6

Grice, H. Paul (1975). "Logic and conversation:. In P. Cole \& J. L. Morgan (Eds.), Syntax and semantics 3 (41-58). New York: Academic Press.

Haspelmath, Martin (2006). "Against markedness (and what to replace it with)". Journal of Linguistics, 43(1), 25-70. DOI: 10.1017/S0022226705003683

Hearst, Marti A. (1992). "Automatic acquisition of hyponyms from large text corpora". Proceedings of the fourteenth international conference on computational linguistics, Nantes, France. DOI: 10.3115/992133.992154

Horn, Laurence R. (1984). "Toward a new taxonomy for pragmatic inference". In D Schiffrin (Ed.), Meaning, form, and use in context (11-42). Washington: Georgetown University Press.

Huang, Yan (2009). "Neo-Gricean pragmatics and the lexicon". International Review of Pragmatics, 1, 118-153. DOI: 10.1163/187731009X455866

Ide, Nancy \& Jean Véronis (1993). "Extracting knowledge bases from machine-readable dictionaries: Have we wasted our time?" Knowledge Bases \& Knowledge Structures 93, Tokyo.

Jones, Steven (2002). Antonymy: A corpus-based perspective. London: Routledge.

Justeson, John S. \& Slava M. Katz (1992) "Redefining antonymy". Literary and Linguistic Computing, 7, 176-184. DOI: 10.1093/llc/7.3.176

Kempson, Ruth M. (1980). “Ambiguity and word meaning”. In S. Greenbaum, G. Leech \& J. Svartvik (Eds.), Studies in English linguistics (7-16). London: Longman.

Langacker, Ronald W. (1987). Foundations of cognitive grammar (Vol. 1). Stanford: Stanford University Press.

Lehrer, Adrienne (1990a). "Prototype theory and its implications for lexical analysis". In S. L. Tsohatzidis (Ed.), Meanings and prototypes (368-381). London: Routledge.

Lehrer, Adrienne (1990b). "Polysemy, conventionality, and the structure of the lexicon". Cognitive Linguistics, 1(2), 207-246. DOI: 10.1515/cogl.1990.1.2.207

Lyons, John (1977). Semantics (Vol. 1). Cambridge: Cambridge University Press.

Manning, Christopher D. \& Hinrich Schütze (1999). Foundations of statistical natural language processing. Cambridge, MA: MIT Press. 
Mettinger, Arthur (1994). Aspects of semantic opposition in English. Oxford: Clarendon Press.

Mititelu, Verginica B. (2006). "Automatic extraction of patterns displaying hyponymhypernym co-occurrence from corpora". Proceedings of the first CESCL. Budapest, Hungary.

Murphy, M. Lynne (2003). Semantic relations and the lexicon. Cambridge: Cambridge University Press.

Oxford English Dictionary, $2^{\text {nd }}$ ed. (1989). Oxford: Oxford University Press.

Quine, Willard van Orman (1960). Word and object. Cambridge, MA: MIT Press.

Rohdenburg, Günther (1985a). "Dogs, bitches and other creatures". Journal of Semantics, 4, 117-135.

Rohdenburg, Günther (1985b). "Unmarked and marked terms in English”. In G. A. J. Hoppenbrouwers, P. A. M. Seuren \& A. J. M. M. Weijters (Eds.), Meaning and the lexicon (63-71). Dordrecht: Forris. DOI: 10.1093/jos/4.2.117

Traugott, Elizabeth Closs \& Richard B. Dasher (2002). Regularity in semantic change. Cambridge: Cambridge University Press.

Tuggy, David (1993). “Ambiguity, polysemy, and vagueness". Cognitive Linguistics, 4(3), 273-290. DOI: 10.1515/cogl.1993.4.3.273

Zgusta, Ladislav (1971). Manual of lexicography. The Hague: Mouton.

Zwicky, Arnold M. \& Jerrold M. Sadock (1975). "Ambiguity tests and how to fail them". In J. P. Kimball (Ed.), Syntax and semantics 4 (1-36). London: Academic Press.

\begin{abstract}
About the author
Anu Koskela received her Doctorate in Linguistics from the University of Sussex, Brighton, UK, and currently works as a Lecturer in English Language at De Montfort University in Leicester, UK. Her research is focused on lexical semantics and cognitive linguistics, particularly on polysemy, categorisation, meaning relations and metonymy. She is the co-author (with M. Lynne Murphy) of Key Terms in Semantics (2010, Continuum).
\end{abstract}

